\title{
Tamoxifen treatment for breast cancer enforces a distinct gene-expression profile on the human endometrium: an exploratory study
}

\author{
S C J P Gielen ${ }^{1}, L C M$ Kühne ${ }^{2}, P C$ Ewing ${ }^{3}, L J$ Blok $^{2}$ and C W Burger ${ }^{1}$
}

\begin{abstract}
${ }^{1}$ Department of Obstetrics and Gynecology, ${ }^{2}$ Department of Reproduction and Development and ${ }^{3}$ Department of Pathology, Erasmus MC University Medical Center Rotterdam, PO Box 1738, 3000 DR Rotterdam, The Netherlands
\end{abstract}

(Requests for offprints should be addressed to L J Blok; Email: I.blok@erasmusmc.nl)

\begin{abstract}
Tamoxifen treatment for breast cancer increases proliferation of the endometrium, resulting in an enhanced prevalence of endometrial pathologies, including endometrial cancer. An exploratory study was performed to begin to understand the molecular mechanism of tamoxifen action in the endometrium. Gene-expression profiles were generated of endometrial samples of tamoxifen users and compared with matched controls. The pathological classification of samples from both groups included atrophic/inactive endometrium and endometrial polyps. Unsupervised clustering revealed that samples of tamoxifen users were, irrespective of pathological classification, fairly similar and consequently form a subgroup distinct from the matched controls. Using SAM analysis (a statistical method to select genes differentially expressed between groups), 256 differentially expressed genes were selected between the tamoxifen and control groups. Upon comparing these genes with oestrogen-regulated genes, identified under similar circumstances, $95 \%$ of the differentially expressed genes turned out to be tamoxifen-specific. Finally, construction of a gene-expression network of the differentially expressed genes revealed that 69 genes centred around five well-known genes: TP53, RELA, MYC, epidermal growth factor receptor and $\beta$-catenin. This could indicate that these well-known genes, and the pathways in which they function, are important for tamoxifencontrolled proliferation of the endometrium.
\end{abstract}

Endocrine-Related Cancer (2005) 12 1037-1049

\section{Introduction}

Tamoxifen is the first-choice adjuvant treatment for primary oestrogen receptor-positive $(\mathrm{ER}+)$ breast cancer in postmenopausal women. It has been shown that survival rates in tamoxifen-treated women are improved as much as $50 \%$ (Early Breast Cancer Trialists' Collaborative Group, 1998). Furthermore, tamoxifen use has also been shown to reduce the incidence of breast cancer in healthy women at high risk for this disease (Powles 1998, Veronesi et al. 1998, Mokbel 2003). The mechanism of action of tamoxifen in breast cancer patients is that it inhibits cancer cell growth by competitive antagonism with oestrogen for its receptor (Ring \& Dowsett 2004).
One of the most significant side effects of treatment with tamoxifen appears to be its proliferative effect on the endometrium (oestrogen-agonistic effect; Buzdar 1998, Bergman et al. 2000). Several studies have evaluated the incidence of endometrial pathologies in tamoxifen users, and although occurrence rates differ per study, a higher incidence in the tamoxifen group is generally agreed on. Endometrial pathologies associated with tamoxifen use include hyperplasia, polyps, carcinomas and sarcomas (Cohen 2004).

The mechanism of action of tamoxifen is very complex. It is generally agreed that conformational change of the receptor after ligand binding differs between oestrogen and tamoxifen, resulting in binding of other co-factors to the ligand-receptor complex 
(Brzozowski et al. 1997). The tissue-dependent mode of action of tamoxifen can then be explained by the relative abundance or paucity of co-factors in different tissues (McDonnell 2004). For example, in breast cells the co-repressors NCoR and SMRT are recruited to the ER-tamoxifen complex, while in endometrial cells the co-activators SRC1, AIB1 and CBP are recruited to the ER-tamoxifen complex (Shang \& Brown 2002, Shang et al. 2000).

The formation of the ER-tamoxifen complex results in downstream activation of genes and pathways. Several genes have been studied to investigate the effects of tamoxifen on the human endometrium. It has been suggested that the expression of transforming growth factor $\beta-1$ (TGF $\beta-1), p 27$, cathepsin $D$ and CA125 is different in benign endometrial samples of tamoxifen users when compared with non-users (Carmichael et al. 2000, Mylonas et al. 2003a, 2003b, Siufi et al. 2003). In contrast, the expression of inhibin/ activin $\alpha$ and $\beta$ in polyps was found to be similar in both groups (Mylonas et al. 2004). Furthermore, the apoptosis/proliferation index, determined by measuring the proliferation marker Ki67 and the apoptosis markers Fas, FasL and Bcl-2, is higher in benign endometria of tamoxifen users compared with endometria of non-users (Mourits et al. 2002a, 2002b).

Interestingly, differences in expression levels of several genes are not observed when endometrial carcinomas from tamoxifen users and matched controls are compared. Microarray analysis has so far not shown any difference in gene-expression profiles between tamoxifen-associated tumours and matched controls (matched for stage, grade and histology; Ferguson et al. 2004). It is true, however, that endometrial tumours seem to develop sooner and are more aggressive in tamoxifen-treated patients (Cohen 2004).

In the current investigations, it was speculated that the effects of tamoxifen on gene expression will be detected specifically in the benign endometria of tamoxifen users. To explore this hypothesis further a genome-wide microarray was used to generate geneexpression profiles of samples containing $100 \%$ endometrium of women exposed to tamoxifen (seven patient samples) in comparison with matched controls (six patient samples). Interestingly, even in this limited patient group, it was observed that the gene-expression profile of endometria obtained from women using tamoxifen was very distinct from endometria of matched controls. Furthermore, upon comparing the differentially expressed genes with oestrogen-regulated genes identified under similar circumstances, most of the differentially expressed genes turned out to be tamoxifen-specific. Finally, to gain insights into the cellular effects of tamoxifen, we generated a geneexpression network, which seems to point to relatively profound effects of tamoxifen on cell-cycle regulation and cell survival.

\section{Materials and methods}

\section{Tumour samples and clinicopathologic characteristics}

The human subjects review board of the Erasmus MC, University Medical Center Rotterdam, The Netherlands approved this study. Between 2002 and 2004 endometrial curettages and endometrial tissues from abdominal uterus extirpations were obtained from patients attending the Gynaecologic Oncology Unit at the Erasmus MC University Medical Center (Department of Obstetrics and Gynaecology) for vaginal bleeding. All patients were postmenopausal, defined as having no menstrual period in the preceding 12 months. There were two patient groups: one group used tamoxifen as adjuvant therapy for breast cancer (tamoxifen users) and the other group (control) had not used tamoxifen or any other sex hormone-related therapy. As patients with node-positive breast tumours and a positive oestrogen and/or progesterone receptor status are all treated with tamoxifen in The Netherlands, it was impossible to fully match the tamoxifenusers group with a control group consisting also of node-positive and receptor-positive breast cancer patients. Therefore, our control group did not contain any breast cancer patients.

Before using any of the surgical specimens, the histological classification of all curettage and hysterectomy specimens was revised using standardized guidelines by a pathologist experienced in gynaecopathology (H A Klaassens, F E van Wijk, P HanifiMoghaddam, unpublished observations). The endometrium was assessed as inactive/atrophic or as containing any proliferative activity, hyperplasia or a polyp. Where the specimen was a curettage, the presence of a polyp was determined by assessment of the form of the fragment, presence of large blood vessels, nature of the stroma and comparison with adjacent endometrium. The appearance of the glands within the polyp was recorded.

In total, primary tissues from 17 patients who had used tamoxifen and eight control patients were collected, and all specimens were snap-frozen and stored at $-80^{\circ} \mathrm{C}$. Sandwich sections were made of the samples to establish the percentage of endometrium (Smid-Koopman et al. 2004). Of the 17 endometrial 
tissues from tamoxifen users, nine samples were excluded from further evaluation: in eight of these samples the amount of endometrial tissue was to small (less than 100\% endometrium) and one sample was classified by histology as endometrial cancer. From the remaining eight samples RNA was isolated and the quality of RNA was verified. In one of these eight samples the RNA turned out to be degraded. The remaining seven samples were used for a microarray experiment.

These seven samples were matched, based on histological classification, to tissue samples of patients attending the hospital for vaginal bleeding but without prior tamoxifen exposure (and without breast cancer). Initially eight control samples were collected, but from one sample the amount of endometrial tissue was to low and one sample was excluded because of malignant pathology. Patients who had used hormonereplacement therapy were excluded from this control group. Patient characteristics of the tamoxifen and control groups were compared using one-way analysis of variance (ANOVA; SPSS version 11).

\section{RNA isolation, amplification and hybridization}

Total RNA was isolated by sonification of the sample in TRIzol buffer (Invitrogen Life Technologies, Carlsbad, CA, USA) for $1 \mathrm{~min}$ at $4{ }^{\circ} \mathrm{C}$ and then purified using RNeasy columns (QIAGEN Benelux BV, Venlo, The Netherlands). Quality of RNA was ensured before labelling by analysing $20 \mathrm{ng}$ of each sample using the RNA 6000 NanoAssay and the Bioanalyzer 2100 (Agilent Technologies Netherlands BV, Amstelveen, The Netherlands). From each sample cRNA was synthesized and labelled according to the Affymetrix protocol, following hybridization to the U133 plus 2.0 GeneChip (Affymetrix, Santa Clara, CA, USA).

\section{Data normalization and analysis}

Raw expression values were analysed using the GeneChip Operating Software (GCOS) version 1.0, provided with the Affymetrix GeneChip service. Intensity values were scaled to an average of 100 per GeneChip according to the method of global scaling provided in the GCOS. Using this method only reliable results are generated for samples with an average intensity value of 30 or more, and therefore all values between 0 and 30 were set at 30 (Valk et al. 2004). This procedure affected $46 \%$ of all intensity values, of which $95 \%$ were flagged as absent or marginal by the GCOS, while $5 \%$ were flagged as present according to the GCOS, indicating the reliability of this method.
The following steps were undertaken to normalize the data, using Microsoft Excel software. As a first step, per gene, the geometric mean of the hybridization intensities over all samples was calculated. Secondly, for all samples the level of expression per gene was determined relative to the geometric mean for that gene. As a last step, the newly generated expression levels were log-transformed (on a base 2 scale) to equally ascribe gene-expression levels with similar relative distance to the geometric mean (up- and downregulation relative to the geometric mean). As a result of this, deviation from the geometrical mean reflects differential gene expression.

\section{Clustering}

Using the Omniviz package (www.omniviz.com), unsupervised cluster analysis was performed (external information such as tamoxifen use and pathological classification were not taken into account). Genes (probesets) whose level of expression was indicated as present (using GCOS) in at least one patient were selected for further analysis; this affected $60 \%$ of genes.

Cluster analysis of genes significantly differentially expressed between the tamoxifen group and the controls was performed with EPSCLUST (expression profile data clustering and analysis) at http://ep.ebi. ac.uk/EP/EPCLUST.

\section{SAM analysis}

Supervised analysis was performed with the use of SAM software provided as a supplement for Microsoft Excel (www-stat.stanford.edu/ tibs/SAM). Using this analysis gene-expression profiles are related to external variables, in this case tamoxifen exposure and histological classification. SAM calculates a score per gene based on the change in expression relative to the standard deviation of all measurements for that gene. The criteria to identify genes assigned to a cluster were: minimal difference in gene expression between the assigned cluster and the other samples by a factor of 2 $\left(\log _{2} \leq 1\right.$, or $\left.\log _{2}>1\right)$, and a $q$ value of less than $5 \%$. The $q$ value is similar to the $P$ value and represents the probability of a falsely assigned differentially expressed gene between clusters.

\section{Biological classification and pathway analysis}

For functional classification of genes significantly differentially expressed between the tamoxifen group and the controls, we used Pathway Assist 2.5 (Ariadne 
Genomics, Rockville, MD, USA). The Pathway Assist database contains biological knowledge represented in a formalized form focused on how proteins, cellular processes and small molecules interact, modify and regulate each other. Pathway Assist provides a method for searching objects individually by keyword, string or attributes. These include, for example, type (protein, enzyme), effect (positive, negative, unknown), mechanism (transcription, phosphorylation), tissue type, biological process, belonging to cell structure and others. The complete databases of the Kyoto Encyclopedia of Genes and Genomes (www.genome.ad.jp/ kegg), Database of Interacting Proteins (DIP; http:// dip.doe-mbi.ucla.edu), Bimolecular Interaction Network Database (BIND; http://bind.mshri.on.ca) and Gene Ontology (GO; www.geneontology.org) were imported into the Pathway Assist database.

For building gene-expression networks, Ingenuity Pathway analysis was used (https://analysis.ingenuity. $\mathrm{com} / \mathrm{pa} /$ ). This database utilizes the Ingenuity Pathway Knowledge Base (IPKB) to computationally analyse datasets to identify networks or pathways.

\section{Quantitative PCR}

Validation of microarray expression data was accomplished by selection of six genes. First-strand cDNA synthesis was performed using $2 \mu \mathrm{g}$ total RNA and the Superscript 2 enzyme (Gibco, Carlsbad, CA, USA) according to a standardized protocol (available on request from L J B; email: 1.blok@erasmusmc.nl). Real-time PCR was performed using the SYBR Green PCR Kit (Applied Biosystems, Foster City, CA, USA) in the Opticon 2 apparatus (MJ Research, Bio-Rad Laboratories, Waltham, MA, USA).

For each sample, $5 \mathrm{ng}$ cDNA was used for the PCR. Per reaction, a melting-curve analysis was performed following each experiment to ensure the presence of a single amplified product. All PCRs were performed in duplicate. The starting quantity for each analysed gene was determined using Opticon monitor software. Using this quantity, the expression level of each gene was normalized to the expression level of the reference gene, $\beta$-actin. One-way ANOVAs were performed to assess $P$ values of differences between the control and tamoxifen groups.

\section{Results}

\section{Patients}

Information about the subjects participating in the study is given in Table 1. All patients were postmenopausal and the mean age for the two patient
Table 1 Clinicopathologic characteristics of tissue samples

\begin{tabular}{lrlc}
\hline Group & $\begin{array}{c}\text { Patient } \\
\text { no. }\end{array}$ & \multicolumn{1}{c}{ Age (years) Pathology } & $\begin{array}{c}\text { Tamoxifen } \\
\text { (months) }\end{array}$ \\
\hline G1 & 43 & 47 Inactive & 27 \\
G1 & 45 & 38 Inactive & 36 \\
G2 & 105 & 57 Polyp, cystic atrophy & 24 \\
G2 & 112 & 57 Polyp, cystic atrophy & 48 \\
G2 & 116 & 55 Polyp, simple & 60 \\
& & hyperplasia without atypia & \\
G2 & 33 & $\begin{array}{l}\text { 87 Polyp, simple } \\
\text { hyperplasia without atypia }\end{array}$ & 24 \\
G2 & 74 & 63 Polyp, largely inactive & 30 \\
& & with focal simple hyperplasia & \\
G3 & 120 & without atypia & 49 Disorded proliferation \\
G3 & 122 & 60 Cystic atrophy & - \\
G3 & 29 & 63 Cystic atrophy & - \\
G4 & 123 & 52 Polyp, cystic atrophy & - \\
G4 & 125 & 61 Polyp, cystic atrophy & - \\
G4 & 44 & 67 Polyp, cystic atrophy & - \\
\hline
\end{tabular}

Details of groups G1-G4 are given in the Results section.

groups was similar $(P=0.891)$ : the mean age in the tamoxifen group was $57.7 \pm 15.3$ years and in the control group was $58.7 \pm 6.9$ years. The median duration of tamoxifen use in the tamoxifen group was 35 months (24-60 months). All patients were referred to a gynaecologist because of vaginal bleeding. The tamoxifen-using patients were all treated because of node-positive breast tumours with a positive oestrogen and/or progesterone receptor status and none of the patients had received chemotherapy. The control patients were matched to the tamoxifen-using patients, but the control group did not contain any breast cancer patients. Furthermore, none of the control patients were using hormones that could affect the endometrium (that is, hormone-replacement therapy of any sort).

\section{Gene-expression profiles are different in tamoxifen users compared with non-users}

Based on histological classification (atrophic/inactive endometrium or endometrial polyp) and whether the patients were exposed to tamoxifen, samples were divided into four groups (Table 1). Group 1 (G1) were samples of atrophic/inactive endometrial tissues from tamoxifen users, group 2 (G2) were polyps from tamoxifen users, group 3 (G3) were samples of atrophic/inactive endometrial tissues from nontamoxifen users and group 4 (G4) were polyps from non-tamoxifen users (Table 1). To test whether the generated gene-expression profiles of all samples reflect 

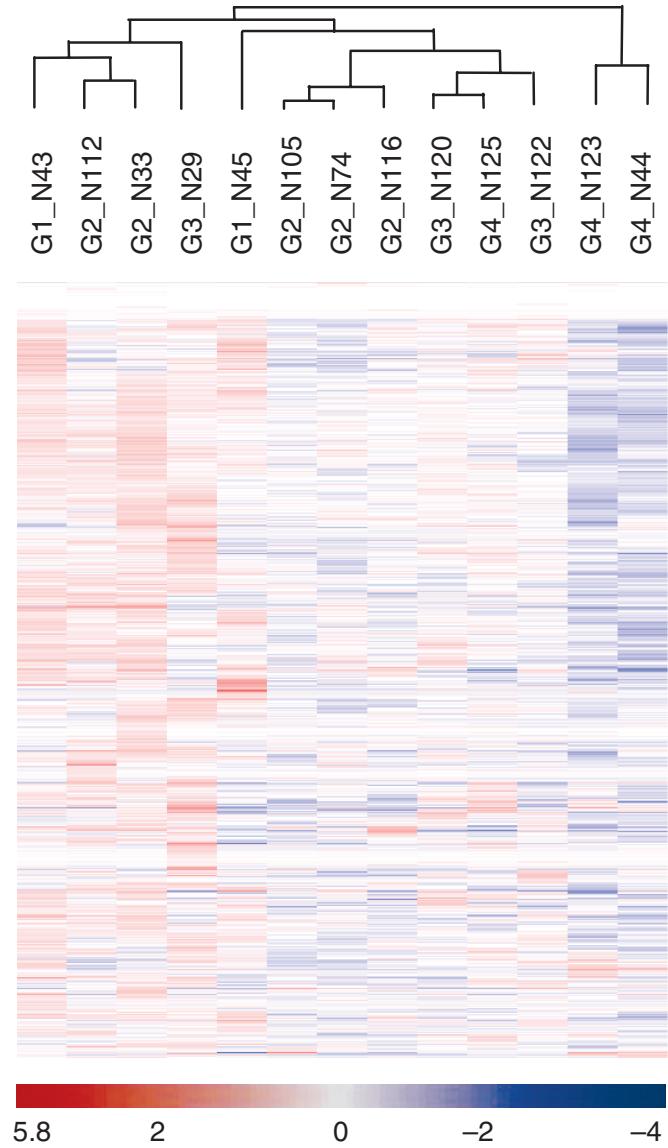

Figure 1 Cluster analysis of gene-expression profiles of tamoxifen users and non-users. Clustering of all genes from all endometrial samples was performed. The expression level of each gene for every patient sample was determined relative to the geometric mean for that gene over all samples. The dendogram at the top illustrates the formation of several arbitrary groups. For each sample the group (G; based on pathology and tamoxifen exposure; see Results section for details) is indicated with the patient number $(\mathrm{N})$.

the classification based on histology and tamoxifen exposure, unsupervised cluster analysis was performed (Fig. 1). In this analysis the samples were clustered into subgroups without taking into account external information. From this analysis it became clear that the samples were clustered into subgroups that reflected their histological classification and exposure to tamoxifen, with the exception of one sample of group 3 (Fig. 1). Furthermore, samples of the tamoxifen-user groups (G1 and G2) cluster were separate from the non-tamoxifen-user groups (G3 and G4). This implicates that the factor of tamoxifen exposure bares much more weight in clustering of the subgroups than does histological classification into atrophic/inactive or polypus endometrium. The complete list of genes can be found at our website: www2.eur.nl/fgg//rede/gielen(expression_data).

\section{Genes differentially expressed between the tamoxifen and control groups}

To identify genes that are related to tamoxifen exposure, samples in G1 and G2 were defined as the tamoxifen group, and samples in G3 and G4 as the control group. SAM analysis was performed between the tamoxifen and control groups, and the genes identified as being differentially regulated between the two groups reflected genes whose expression is affected by tamoxifen exposure.

SAM analysis revealed that the hybridization signal intensity of 256 genes in the tamoxifen group were either 2-fold up- or 2-fold downregulated compared with the control group. The fold differences are the average ratios resulting from consistent changes between the tamoxifen and control groups. Unsupervised clustering was performed for these 256 differentially expressed genes (Fig. 2) and, as expected, two major clusters separating the tamoxifen and control groups were formed.

Of the 256 genes, 227 were known, whereas the others represented expressed sequence tags (ESTs). Some of these genes and their roles in endometrial functioning have been described before (HOXB7 and HOXA5 (Yanaihara et al. 2004); SLP1 (Zhang et al. 2002) and hepatocyte growth factor receptor (Khan et al. 2003, Yoshida et al. 2004)), but for most genes this is a new finding. Furthermore, several genes were earlier linked to the ER, for example EVA1 and TPD52L1 (Gielen et al. 2005b). The complete list of differentially expressed genes can be found at www2.eur.n1/fgg//rede/gielen (SAM analysis).

\section{Biological classification of regulated genes}

The discriminative genes identified with SAM analysis may reveal functional pathways that are critical for tamoxifen-induced endometrial pathology. As a first step towards investigating this, we studied which biological processes these genes affect. Using the Pathways Assist database, which combines a number of other databases (such as the GO and KEGG databases), several functional categories were identified. Out of the 227 known genes, 85 could be classified and, interestingly, most of the genes were involved in proliferation (39 genes), apoptosis (27 genes) and/or differentiation (27 genes). The complete list of biological processes can be found at http://www2.eur.nl/ $\mathrm{fgg} / /$ rede/gielen(biological classification). 


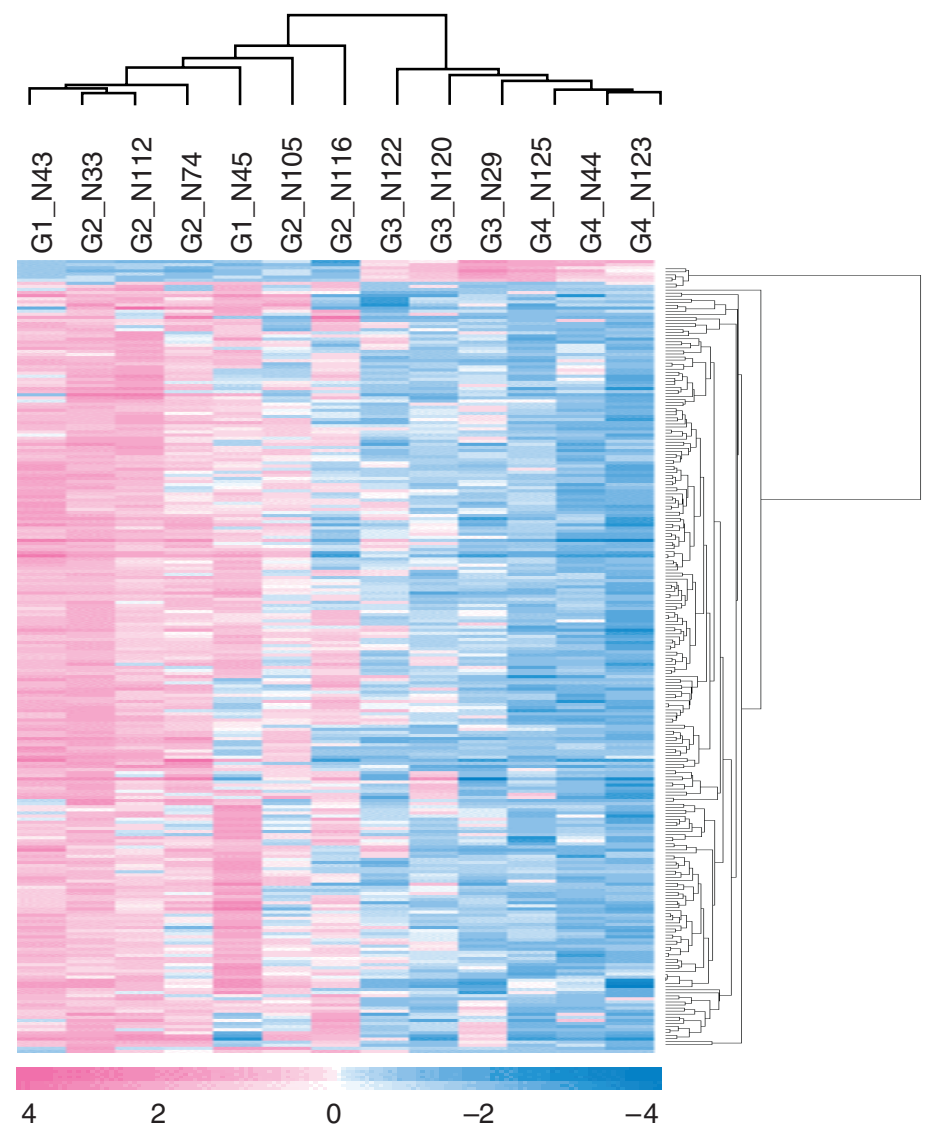

Figure 2 Genes differentially expressed between the two groups. Hierarchical clustering of genes differentially expressed between the tamoxifen and control groups. Genes were selected to be differentially expressed using SAM analysis if $q<0.05$ and fold induction was $>2$. The dendogram at the top illustrates the formation of several arbitrary groups, and the dendogram on the right illustrates the different gene clusters. For each sample the group (G; based on pathology and tamoxifen exposure; see Results section for details) is indicated with the patient number $(\mathrm{N})$.

\section{Regulation of signalling pathways}

Thus far we have shown that the genes that were differentially regulated between the tamoxifen and control groups modulate several biological processes. This led us to question whether these genes belonged to the same signalling networks. In other words, what is the interrelationship between the regulated genes, and in which canonical pathways do they lie? To study this question, gene-expression networks were constructed using the Ingenuity database. In such a geneexpression (biological) network, molecules are nodes, and different types of connection represent interactions between the different genes. It should be emphasized that this 'network analysis' is an exploratory in silico approach and so is only a model of a biological pathway and does not indicate that the pathway or network actually exists.
The 227 known genes from the 256 genes found to be differentially expressed between the groups were used for this analysis. Using the Ingenuity database, 86 out of these 227 known genes could be clustered into 23 networks. Six of these networks contained more than one regulated gene and Fig. 3 displays a compilation of these six networks. In Table 2 the genes acting in these networks are listed. Three of the six networks could be merged and are shown as network 1 in Fig. 3. This network centres on cyclindependent kinase inhibitor 1A (CDKN1A), $\beta$-catenin (CTNNB1) and tumour protein p53 (TP53). Network 2 centres around $\mathrm{v}$-Myc myelocytomatosis viral oncogene homologue (MYC) and network 3 around $\mathrm{v}-\mathrm{Rel}$ reticuloendotheliosis viral oncogene homologue $\mathrm{A}$ or nuclear factor- $\kappa \mathrm{B}(\mathrm{NF}-\kappa \mathrm{B})$ transcription factor subunit p65 (RELA). For network 4 it is less clear, but genes seem to cluster around the epidermal growth 


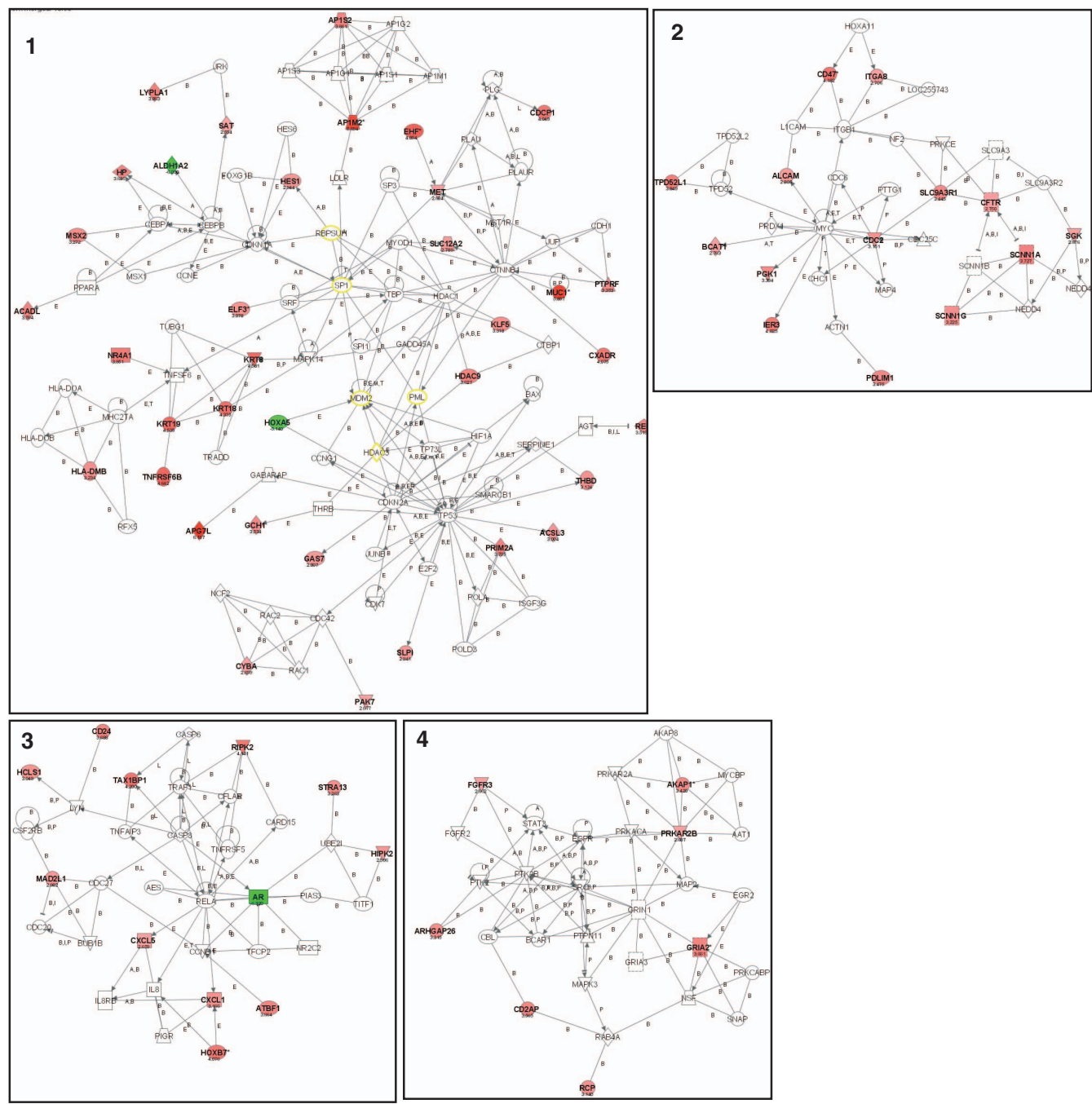

Figure 3 Signalling pathways involved in tamoxifen response. Gene-expression networks were constructed, using the Ingenuity database, for genes differentially expressed between the tamoxifen and control groups. Of the 23 networks, six contain more then one regulated gene. Three of the six networks could be merged (network 1), and centre around CDKN1A, CTNNB1 and TP53. The second network (2) centres around MYC, third network (3) around RELA and the fourth (4) network around the EGF receptor.

factor receptor (EGF receptor). For reasons of clarity, several expressed but non-regulated genes were also included in the network analysis (non-coloured blocks). Results from the network analysis are evaluated further in the Discussion section.

\section{Validation of microarray data}

Two kinds of validation were performed: an analysis of profile reproducibility and a verification of microarray expression data.

Profile reproducibility was analysed for RNA isolated from two postmenopausal endometria of non-treated patients. From both patients two $1 \mu \mathrm{g}$
RNA samples were used. From each $1 \mu \mathrm{g}$ sample, cRNA was synthesized and labelled according to the Affymetrix protocol, following hybridization to the U133 plus 2.0 GeneChip. Generation of cRNA, labelling and hybridizations were all performed independently several weeks apart from each other. Upon reviewing the results, it was observed that the false discovery rates were 1.6 and $1.1 \%$, respectively. These percentages indicate that the technical reproducibility of the microarray experiments was very high.

As a second step to verifying our microarray data, expression of a number of genes was measured using real-time PCR. The genes KRT18, AR, TGF $\beta-1$, 
Table 2 Genes differentially expressed between the tamoxifen and control groups and assigned to the generated networks All other genes can be found on our website www2.eur.nl/fgg//rede/gielen.

\begin{tabular}{|c|c|c|c|c|c|}
\hline $\begin{array}{l}\text { Gene } \\
\text { symbol }\end{array}$ & Gene title & GenBank ID & UniGene ID & Fold change & $q$ value \\
\hline ACADL & acyl-coenzyme A dehydrogenase, long chain & Al367275 & Hs.430108 & 2.107525975 & 0.028968 \\
\hline ACSL3 & acyl-CoA synthetase long-chain family member 3 & AL525798 & Hs.268012 & 2.030415626 & 0.036416 \\
\hline AKAP1 & A kinase (PRKA) anchor protein 1 & BC000729 & Hs.78921 & 2.374450908 & 0.035784 \\
\hline ALCAM & activated leukocyte adhesion molecule & AA156721 & Hs.150693 & 0.4909 & 0.036416 \\
\hline ALDH1A2 & aldehyde dehydrogenase 1 family, member $\mathrm{A} 2$ & NM_003888 & Hs.435689 & -2.9995 & 0.020632 \\
\hline $\mathrm{AP} 1 \mathrm{M} 2$ & adaptor-related protein complex 1 , mu 2 subunit & NM_005498 & Hs.18894 & 2.361832782 & 0.017815 \\
\hline AP1S2 & $\begin{array}{l}\text { adaptor-related protein complex } 1 \text {, sigma } 2 \\
\text { subunit }\end{array}$ & AA205444 & Hs.121592 & 0.44094 & 0.022774 \\
\hline APG7L & APG7 autophagy 7-like (S. cerevisiae) & BC000091 & Hs.38032 & 2.022040239 & 0.017815 \\
\hline AR & androgen receptor & AF162704 & Hs.99915 & -2.11902 & 0.020632 \\
\hline ARHGAP26 & Rho GTPase activating protein 26 & Al768563 & Hs.132942 & 2.458331285 & 0.028428 \\
\hline ATBF1 & AT-binding transcription factor 1 & NM_006885 & Hs.108806 & 2.364457475 & 0.028968 \\
\hline BCAT1 & branched chain aminotransferase 1 , cytosolic & AL390172 & Hs.438993 & 2.908583229 & 0.046272 \\
\hline CD24 & $\begin{array}{l}\text { CD24 antigen (small cell lung carcinoma cluster } 4 \\
\text { antigen) }\end{array}$ & M58664 & Hs.375108 & 2.129970819 & 0.028968 \\
\hline CD2AP & CD2-associated protein & NM_012120 & Hs.374340 & 2.082509007 & 0.028968 \\
\hline CD47 & $\begin{array}{l}\text { CD47 antigen (Rh-related antigen, integrin- } \\
\text { associated signal transducer) }\end{array}$ & BG230614 & Hs. 446414 & 2.348520432 & 0.020632 \\
\hline CDC2 & cell division cycle $2, \mathrm{G} 1$ to $\mathrm{S}$ and $\mathrm{G} 2$ to $\mathrm{M}$ & D88357 & Hs.334562 & 2.103 & 0.035784 \\
\hline CDCP1 & CUB domain-containing protein 1 & NM_022842 & Hs. 146170 & 2.146153021 & 0.018863 \\
\hline CFTR & $\begin{array}{l}\text { cystic fibrosis transmembrane conductance } \\
\text { regulator }\end{array}$ & NM_000492 & Hs.411882 & 2.827494557 & 0.039344 \\
\hline CXADR & coxsackie virus and adenovirus receptor & NM_001338 & Hs.79187 & 2.170609941 & 0.027545 \\
\hline CXCL1 & chemokine (C-X-C motif) ligand 1 & NM_001511 & Hs.789 & 7.175660161 & 0.035784 \\
\hline CXCL5 & chemokine ( $\mathrm{C}-\mathrm{X}-\mathrm{C}$ motif) ligand 5 & AK026546 & Hs. 89714 & 9.875567845 & 0.041008 \\
\hline CYBA & cytochrome b-245, alpha polypeptide & NM_000101 & Hs.68877 & 2.008516108 & 0.041008 \\
\hline $\mathrm{EHF}$ & Ets homologous factor & Al763378 & Hs.200228 & 2.216017374 & 0.017815 \\
\hline ELF3 & $\begin{array}{l}\text { E74-like factor } 3 \text { (ets domain transcription factor, } \\
\text { epithelial-specific) }\end{array}$ & U73844 & Hs.67928 & 2.237036374 & 0.036416 \\
\hline FGFR3 & fibroblast growth factor receptor 3 & NM_000142 & Hs. 1420 & 2.0702 & 0.041008 \\
\hline GAS7 & growth arrest-specific 7 & BC001152 & Hs.462214 & 2.084810074 & 0.036416 \\
\hline $\mathrm{GCH} 1$ & $\begin{array}{l}\text { GTP cyclohydrolase } 1 \text { (dopa-responsive } \\
\text { dystonia) }\end{array}$ & NM_000161 & Hs. 86724 & 2.012355865 & 0.028968 \\
\hline GRIA2 & glutamate receptor, ionotropic, AMPA 2 & BE219628 & Hs.33 & 5.0 & 2774 \\
\hline HCLS1 & hematopoietic cell-specific Lyn substrate 1 & NM_005335 & Hs. 14601 & 2.248858704 & 0.036416 \\
\hline HDAC9 & histone deacetylase 9 & NM_014707 & Hs.487662 & 2.35977063 & 0.028968 \\
\hline HES1 & hairy and enhancer of split $1,($ Drosophila) & NM_005524 & Hs.250666 & 2.089252883 & 0.036416 \\
\hline HIPK2 & Homeodomain interacting protein kinase 2 & BF218115 & Hs.397465 & 2.538908777 & 0.0372 \\
\hline HLA-DMB & $\begin{array}{l}\text { major histocompatibility complex, class II, } \\
\text { DM beta }\end{array}$ & NM_002118 & Hs.1162 & 2.393260578 & 0.035784 \\
\hline HOXA5 & homeo box A5 & NM_019102 & Hs.37034 & -4.82313 & 0.033765 \\
\hline HOXB7 & homeo box B7 & S49765 & Hs. 436181 & 2.171081198 & 0.027545 \\
\hline HP & haptoglobin & NM_005143 & Hs. 403931 & 3.062411956 & 0.028968 \\
\hline IER3 & immediate early response 3 & NM_003897 & Hs.76095 & 4.072158651 & 0.027545 \\
\hline ITGA8 & integrin, alpha 8 & Al193623 & Hs. 171025 & 2.030333178 & 0.039344 \\
\hline KLF5 & Kruppel-like factor 5 (intestinal) & $\mathrm{AF} 132818$ & Hs. 84728 & 2.276659685 & 0.033765 \\
\hline KRT18 & keratin 18 & NM_000224 & Hs.406013 & 2.208041688 & 0.020632 \\
\hline KRT19 & keratin 19 & NM_002276 & Hs.309517 & 3.054927598 & 0.020632 \\
\hline KRT8 & keratin 8 & U76549 & Hs.356123 & 2.115864754 & 0.020632 \\
\hline LYPLA1 & lysophospholipase I & BG288007 & Hs.446676 & 2.297371807 & 0.028968 \\
\hline MAD2L1 & MAD2 mitotic arrest deficient-like 1 (yeast) & NM_002358 & Hs.79078 & 2.217196577 & 0.036416 \\
\hline MET & $\begin{array}{l}\text { met proto-oncogene (hepatocyte growth factor } \\
\text { receptor) }\end{array}$ & BG170541 & Hs.419124 & 2.224446669 & 0.041008 \\
\hline MSX2 & msh homeo box homolog 2 (Drosophila) & D89377 & Hs.89404 & 2.602269179 & 0.035784 \\
\hline MUC1 & mucin 1 , transmembrane & NM_002456 & Hs.89603 & 2.786524368 & 0.020632 \\
\hline NR4A1 & nuclear receptor subfamily 4 , group A, member 1 & D85245 & Hs. 1119 & 2.227270702 & 0.027545 \\
\hline
\end{tabular}




\begin{tabular}{|c|c|c|c|c|c|}
\hline $\begin{array}{l}\text { Gene } \\
\text { symbol }\end{array}$ & Gene title & GenBank ID & UniGene ID & Fold change & $q$ value \\
\hline PAK7 & p21(CDKN1A)-activated kinase 7 & AB040812 & Hs.32539 & 2.983560581 & 0.041008 \\
\hline PDLIM1 & PDZ and LIM domain 1 (elfin) & BC000915 & Hs.75807 & 2.013449845 & 0.028968 \\
\hline PGK1 & phosphoglycerate kinase 1 & AA069778 & Hs.78771 & 2.33628484 & 0.026722 \\
\hline PRIM2A & primase, polypeptide $2 \mathrm{~A}, 58 \mathrm{kDa}$ & NM_000947 & Hs.440603 & 2.169762194 & 0.028968 \\
\hline PRKAR2B & $\begin{array}{l}\text { protein kinase, cAMP-dependent, regulatory, } \\
\text { type II, beta }\end{array}$ & NM_002736 & Hs.77439 & 2.030992952 & 0.041008 \\
\hline PTPRF & protein tyrosine phosphatase, receptor type, F & NM_002840 & Hs.75216 & 2.164877035 & 0.033765 \\
\hline $\mathrm{RCP}$ & RAB11 family interacting protein 1 (class I) & NM_025151 & Hs.96125 & 0.45633 & 0.029329 \\
\hline REN & renin & NM_000537 & Hs.3210 & 14.94991778 & 0.028968 \\
\hline RIPK2 & receptor-interacting serine-threonine kinase 2 & AF027706 & Hs. 103755 & 2.104421389 & 0.027545 \\
\hline SAT & spermidine/spermine $\mathrm{N} 1$-acetyltransferase & BE971383 & Hs.28491 & 2.2573873 & 0.049421 \\
\hline SCNN1A & sodium channel, nonvoltage-gated 1 alpha & NM_001038 & Hs.130989 & 2.395783421 & 0.028968 \\
\hline SCNN1G & sodium channel, nonvoltage-gated 1 , gamma & Al985987 & Hs. 145645 & 2.499625056 & 0.029329 \\
\hline SGK & serum/glucocorticoid regulated kinase & NM_005627 & Hs.296323 & 3.082899158 & 0.041008 \\
\hline SLC12A2 & $\begin{array}{l}\text { solute carrier family } 12 \text { (sodium/potassium/ } \\
\text { chloride transporters), member } 2\end{array}$ & AK025062 & Hs.110736 & 2.147996993 & 0.046272 \\
\hline SLC9A3R1 & $\begin{array}{l}\text { solute carrier family } 9 \text { (sodium/hydrogen } \\
\text { exchanger), isoform } 3 \text { regulator } 1\end{array}$ & NM_004252 & Hs.396783 & 4.064544974 & 0.028968 \\
\hline SLPI & $\begin{array}{l}\text { secretory leukocyte protease inhibitor } \\
\text { (antileukoproteinase) }\end{array}$ & NM_003064 & Hs.251754 & 2.319808848 & 0.036416 \\
\hline STRA13 & stimulated by retinoic acid 13 & U95006 & Hs.37616 & 0.34603 & 0.035784 \\
\hline TAX1BP1 & $\begin{array}{l}\text { Tax1 (human T-cell leukemia virus type I) binding } \\
\text { protein } 1\end{array}$ & Al935415 & Hs.5437 & 2.080169742 & 0.020632 \\
\hline THBD & thrombomodulin & NM_000361 & Hs.2030 & 2.185314685 & 0.035784 \\
\hline TNFRSF6B & $\begin{array}{l}\text { tumor necrosis factor receptor superfamily, } \\
\text { member } 6 b\end{array}$ & NM_003823 & Hs.434878 & 2.543687839 & 0.020632 \\
\hline TPD52L1 & tumor protein D52-like 1 & AF208012 & Hs. 16611 & 2.433030827 & 0.028968 \\
\hline
\end{tabular}

CTSD and MUC-1 all showed expression corresponding to our original microarray data (Fig. 2). In the real-time PCR data, expression of the EGF receptor ligand amphiregulin (AREG) was increased in the tamoxifen group compared with the control group, whereas in the microarray data no difference was seen between the two groups (Table 3).

\section{Discussion}

When tamoxifen-induced cancers are matched (for grade and stage) to non-tamoxifen-associated endometrial cancers, no differences are observed in the gene-expression profile (Ferguson et al. 2004). It is only when developing tumours are followed in time that it becomes clear that tamoxifen-induced tumours grow more aggressively than non-tamoxifen-associated endometrial tumours (Cohen 2004). The hypothesis that we chose to start investigating was that tamoxifen specifically induces early endometrial changes leading to a more aggressive cancer phenotype. Therefore, as a first step, gene-expression profiles were generated of early benign endometrial pathology samples of women using tamoxifen, and were compared with geneexpression profiles measured in the same kind of tissue pathology from women not using tamoxifen.

Unsupervised clustering of all genes in all samples revealed that samples of patients who had used tamoxifen clustered together and away from samples of women who had never used tamoxifen. This was somewhat surprising because in both groups atrophic/ inactive as well as endometrial polyps were included. It might have been expected that initially the polyps would cluster together and away from the atrophic/ inactive samples regardless of tamoxifen treatment.

To further investigate the working mechanism of tamoxifen, differentially expressed genes between the tamoxifen and control groups were identified using SAM analysis. Using this method, 256 genes were found specifically regulated by tamoxifen in our patient population. Interestingly, of these 256 genes most were expressed more highly in the tamoxifen group. Since genes can have either a suppressing or an inducing effect on a biological process, this does not necessarily mean that cells in the samples of the tamoxifen group are more biological active. 
Table 3 Validation of differential gene expression by quantitative real-time PCR Data represent the mean ( \pm S.E.) levels per group. Differences between control and treatments were considered significant at $P<0.05$.

\begin{tabular}{|c|c|c|c|c|c|c|}
\hline \multirow[b]{2}{*}{ Gene } & \multicolumn{3}{|c|}{ Microarray } & \multicolumn{3}{|c|}{ Red-time PCR } \\
\hline & Control group & Tamoxifen group & $P$ value & Control group & Tamoxifen group & $P$ value \\
\hline KRT18 & $1( \pm 0.23)$ & $2.21( \pm 0.9)$ & 0.009 & $1( \pm 0.56)$ & $4.9( \pm 3.4)$ & 0.018 \\
\hline $\mathrm{AR}$ & $1( \pm 0.29)$ & $0.5( \pm 0.14)$ & 0.004 & $1( \pm 1.2)$ & $0.25( \pm 0.31)$ & 0.153 \\
\hline AREG & $1( \pm 0.26)$ & $1.21( \pm 0.53)$ & 0.419 & $1( \pm 0.7)$ & $4.7( \pm 3)$ & 0.01 \\
\hline TGFB-1 & $1( \pm 0.4)$ & $0.8( \pm 0.4)$ & 0.444 & $1( \pm 1.1)$ & $0.8( \pm 0.6)$ & 0.672 \\
\hline CTSD & $1( \pm 0.5)$ & $1.5( \pm 0.8)$ & 0.223 & $1( \pm 0.6)$ & $0.9( \pm 0.65)$ & 0.789 \\
\hline MUC-1 & $1( \pm 0.3)$ & $2.8( \pm 0.9)$ & 0.001 & $1( \pm 0.7)$ & $3.6( \pm 2)$ & 0.01 \\
\hline
\end{tabular}

Our next step was to test whether and to what extent the mechanism of action of tamoxifen differs from that of oestrogen. Therefore, the 256 tamoxifen-regulated genes were compared with oestrogen-regulated genes identified from a similar patient population using exactly the same microarray. The patient details will be described in a forthcoming publication $(\mathrm{H} \mathrm{A}$ Klaassens, F E van Wijk, P Hanifi-Moghaddam, unpublished observations), as will data on oestrogenregulated genes (P Hanifi-Moghaddam, B BoersSijmons, H A Klaassens et al., unpublished observations). In short, in these studies postmenopausal women were treated for 3 weeks with oestrogen and compared with matched controls (H A Klaassens, F E van Wijk, P Hanifi-Moghaddam, unpublished observations). Using SAM analysis 746 genes were found differentially expressed between the control group and the oestrogen-treated group. Interestingly, of those 746 genes only 11 overlapped with the 256 genes induced by tamoxifen. As indicated in Fig. 1 this seems to point to a distinct working mechanism of tamoxifen. In cancer cell lines of breast (Frasor et al. 2004), bone (Kian Tee et al. 2004) and endometrium (Gielen et al. 2005a) the unique gene-expression profile after treatment with tamoxifen has been described previously; however, to our knowledge this is the first report of this finding in human benign endometrial samples.

When we subsequently evaluated the function of the tamoxifen-regulated genes, most of these genes were found to be involved in proliferation, apoptosis and differentiation. Upon more thoroughly analysing the biological functions of the tamoxifen-regulated genes it was observed that 69 tamoxifen-regulated genes could be connected with each other in four gene-expression networks. This implies an interrelationship between the regulated genes, and could indicate that several different pathways are orchestrated by tamoxifen signalling in the human endometrium.
An interesting question that can be asked is whether these four gene-expression networks point to a similar physiological function of tamoxifen signalling or whether these networks represent different cellular entities. Since four well-known genes ( $\beta$-catenin, TP53, MYC and RELA) are at the centre of three of the networks and the fourth network more diffusely focuses on growth factor signalling centred on the EGF receptor, the following discussion will concentrate on signalling in the endometrium around these five genes: $\beta$-catenin, TP53, MYC, RELA and EGF receptor.

\section{$\beta$-Catenin}

$\beta$-Catenin (network 1; Fig. 3) has a dual function in the cell. Together with $\alpha$-catinin it links the cell-cell adhesion molecule E-cadherin to the cytoskeleton, and thus stabilizes cell-cell adhesion (Beavon 2000). The other function of $\beta$-catenin is in the canonical Wnt signal transduction pathway (Bienz 2002). In short, Wnt signalling has a central function in the maintenance and control of stem cell compartments where the fine balance between proliferation (Wnt-on) and differentiation (Wnt-off) is regulated (Giles et al. 2003, Logan \& Nusse 2004). In this study $\beta$-catenin itself is not differentially expressed, but this can be explained by the fact that activation through translocation of $\beta$-catenin to the membrane or the nucleus will not be detected in the method used in this study.

The finding that tamoxifen enhances the expression of MUC-1 (increased in breast cancer metastasis; Schroeder et al. 2003), PTPRF (enhanced in metastatic breast cancer; Levea et al. 2000) and CXADR (inhibitor of cancer cell migration; Walters et al. 2002), and the fact that all three genes can bind to $\beta$-catenin, could indicate a specific role of tamoxifen in cell-cell adhesion. The finding that MET expression was enhanced by tamoxifen seems to indicate that Wnt 
signalling is enhanced by tamoxifen (c-MET has recently been identified as a Wnt-regulated gene; Boon et al. 2002). If it is true that the Wnt signal transduction pathway is activated, this is of interest because its central role in homeostasis of adult stem cell niches is reflected by the frequent association of Wnt signalling activating defects in different cancer types, including endometrial cancer (Saegusa et al. 2001, Giles et al. 2003).

\section{Balance between proliferation and apoptosis via the MYC, RELA and TP53 network}

The balance between cell proliferation and cell death is important in epithelial homeostasis. Interestingly, three of the generated gene-expression networks centre on proteins involved in this balance between proliferation and apoptosis (MYC, RELA and TP53). TP53 is a transcription factor and induces a $G_{1}$ arrest in the cell cycle, creating extra time for DNA-repair mechanisms. If DNA repair fails TP53 initiates apoptosis via activation of members of the Bax/Bcl-2 family (Lane $\&$ Fischer 2004). Initiation of apoptosis via activation of the Bax/Bcl-2 family is inhibited by RELA (p65) in complex with p50 (NF- $\kappa \mathrm{B}$ complex; Shukla \& Gupta 2004). The MYC protein is a transcription factor that regulates the cell cycle via regulation of $\mathrm{E} 2 \mathrm{~F}$, cyclin D1 and p27 (progression of the cell cycle), or via activation of TP53 (inhibition of the cell cycle through induction of apoptosis; Patel et al. 2004). In the current investigations, several tamoxifen-regulated genes were found to be connected to TP53, MYC and RELA signalling, suggesting that these networks may be of specific importance for regulation of the endometrial response to tamoxifen. Furthermore, as TP53, MYC and RELA are also themselves interconnected, a putative tamoxifen-induced imbalance in these pathways could play a determining role in endometrial carcinogenesis in tamoxifen users.

\section{EGF receptor signalling}

Earlier work of our group indicated that EGF receptor signalling in the ECC-1 endometrial carcinoma cell line was very important for oestrogen as well as tamoxifen signalling. Furthermore, the EGF receptor ligand amphiregulin (AREG) turned out to be upregulated by oestrogens as well as tamoxifen whereas AREG was also shown to be able to induce growth of the endometrial carcinoma cell line (Gielen et al. 2005a). Also, in the current real-time PCR data AREG was observed to be more highly expressed in the tamoxifen group than the control group (Table 3). The fact that we did not extract this finding from the microarray experiments may be due to the low expression of AREG in the endometrial samples (real-time PCR is more sensitive then microarray analysis). This further strengthens the earlier observation that AREG activation of the EGF receptor may play a role in tamoxifen-induced endometrial pathology. Furthermore, as was also observed earlier in the cell-line experiments (Gielen et al. 2005a), tamoxifen seems to have its own specific effect on some other proteins involved in EGF receptor signalling (as indicated in network 4).

\section{Concluding remarks}

Tamoxifen seems to exert a specific effect on the nonmalignant human endometrium that is different from the effect of oestrogens. As is shown in the current exploratory study, tamoxifen treatment seems to affect several genes involved in proliferation, cell survival, apoptosis, differentiation and cell-cell adhesion of normal endometrial cells. It is of interest to note that the currently available parameters (such as vaginal bleeding, endometrial thickness and dose and duration of tamoxifen use) are not fully associated with endometrial pathology in tamoxifen users (Cohen 2004). Therefore, beginning to understand the molecular mechanism of tamoxifen-induced stimulation of the endometrium is important and maybe a starting point to providing further insights into the early detection of endometrial aberrations that may eventually result in tumour formation. However, additional research with higher patient numbers is needed into those early endometrial changes that may lead to tumour formation.

\section{Acknowledgements}

We are grateful to the medical staff of the Department of Obstetrics and Gynecology and Department of Pathology (Erasmus MC, Rotterdam, The Netherlands) and to the participating women for the gifts of endometrial samples. We thank Wilfred Van IJcken and members of his laboratory from the Center for Biomics, Erasmus MC, for providing the Affymetrix platform. In addition, we thank the Center for Biomics for facilitating BioAnalyzer RNA analysis. We would like to thank E. Lodder (Department of Clinical Genetics) for support with the Omniviz software. The authors declare that there is no conflict of interest that would prejudice the impartiality of this scientific work.

\section{References}

Beavon IR 2000 The E-cadherin-catenin complex in tumour metastasis: structure, function and regulation. European Journal of Cancer 36 1607-1620. 
Bergman L, Beelen ML, Gallee MP, Hollema H, Benraadt J \& van Leeuwen FE 2000 Risk and prognosis of endometrial cancer after tamoxifen for breast cancer. Comprehensive Cancer Centres' ALERT Group. Assessment of Liver and Endometrial cancer Risk following Tamoxifen. Lancet 356 881-887.

Bienz M 2002 The subcellular destinations of APC proteins. Nature Reviews in Molecular \& Cell Biology 3 328-338.

Boon EM, van der Neut R, van de Wetering M, Clevers H \& Pals ST 2002 Wnt signaling regulates expression of the receptor tyrosine kinase met in colorectal cancer. Cancer Research 62 5126-5128.

Brzozowski AM, Pike AC, Dauter Z, Hubbard RE, Bonn T, Engstrom O, Ohman L, Greene G, Gustafsson JA \& Carlquist M 1997 Molecular basis of agonism and antagonism in the oestrogen receptor. Nature $\mathbf{3 8 9}$ 753-758.

Buzdar A 1998 The place of chemotherapy in the treatment of early breast cancer. British Journal of Cancer $\mathbf{7 8}$ (Suppl 4) 16-20.

Carmichael PL, Pole JC \& Neven P 2000 Modulation of endometrial transforming growth factor beta (TGFbeta) by tamoxifen. European Journal of Cancer 36 (Suppl 4) S42-S43.

Cohen I 2004 Endometrial pathologies associated with postmenopausal tamoxifen treatment. Gynecology \& Oncology 94 256-266.

Early Breast Cancer Trialists' Collaborative Group 1998 Tamoxifen for early breast cancer: an overview of the randomised trials. Lancet 351 1451-1467.

Ferguson SE, Olshen AB, Viale A, Awtrey CS, Barakat RR \& Boyd J 2004 Gene expression profiling of tamoxifenassociated uterine cancers: evidence for two molecular classes of endometrial carcinoma. Gynecology \& Oncology 92 719-725.

Frasor J, Stossi F, Danes JM, Komm B, Lyttle CR \& Katzenellenbogen BS 2004 Selective estrogen receptor modulators: discrimination of agonistic versus antagonistic activities by gene expression profiling in breast cancer cells. Cancer Research 64 1522-1533.

Gielen SCJP, Burger CW, Kuhne LCM, Hanifi-Moghaddam P \& Blok LJ 2005a Analysis of estrogen-agonism and -antagonism of tamoxifen, raloxifene and ICI182780 in endometrial cancer cells: a putative role for the EGF receptor ligand amphiregulin. Journal of the Society for Gynecological Investigatoins 12 55-67.

Gielen SCJP, Hanekamp EE, Hanifi-Moghaddam P, Sijbers AM, van Gool AJ, Burger CW, Blok LJ \& Huikeshoven FJM $2005 b$ Growth regulation and transcriptional activities of estrogen and progesterone in human endometrial cancer cells. International Journal of Gynecological Cancer (in press).

Giles RH, van Es JH \& Clevers H 2003 Caught up in a Wnt storm: Wnt signaling in cancer. Biochimica et Biophysica Acta 1653 1-24.

Khan KN, Masuzaki H, Fujishita A, Kitajima M, Sekine I \& Ishimaru T 2003 Immunoexpression of hepatocyte growth factor and c-Met receptor in the eutopic endometrium predicts the activity of ectopic endometrium. Fertility and Sterility 79 173-181.

Kian Tee M, Rogatsky I, Tzagarakis-Foster C, Cvoro A, An J, Christy RJ, Yamamoto KR \& Leitman DC 2004 Estradiol and selective estrogen receptor modulators differentially regulate target genes with estrogen receptors alpha and beta. Molecular Biology of the Cell $\mathbf{1 5}$ 1262-1272.

Lane DP \& Fischer PM 2004 Turning the key on p53. Nature 427 789-790.

Levea CM, McGary CT, Symons JR \& Mooney RA 2000 PTP LAR expression compared to prognostic indices in metastatic and non-metastatic breast cancer. Breast Cancer Research \& Treatment 64 221-228.

Logan CY \& Nusse R 2004 The Wnt signaling pathway in development and disease. Annual Reviews in Cell Development \& Biology 20 781-810.

McDonnell DP 2004 The molecular determinants of estrogen receptor pharmacology. Maturitas 48 (Suppl 1) S7-S12.

Mokbel K 2003 Risk-reducing strategies for breast cancer a review of recent literature. International Journal of Fertility \& Womens Medicine 48 274-277.

Mourits MJ, Hollema H, De Vries EG, Ten Hoor KA, Willemse PH \& Van Der Zee AG 2002a Apoptosis and apoptosis-associated parameters in relation to tamoxifen exposure in postmenopausal endometrium. Human Pathology 33 341-346.

Mourits MJ, Ten Hoor KA, van der Zee AG, Willemse PH, de Vries EG \& Hollema H $2002 b$ The effects of tamoxifen on proliferation and steroid receptor expression in postmenopausal endometrium. Journal of Clinical Pathology 55 514-519.

Mylonas I, Makovitzky J, Richter DU, Jeschke U, Briese V \& Friese K 2003a Cathepsin D expression in normal, hyperplastic and malignant endometrial tissue: an immunohistochemical analysis. Acta Histochemistry 105 245-252.

Mylonas I, Makovitzky J, Richter DU, Jeschke U, Briese V \& Friese K 2003b Immunohistochemical expression of the tumour marker CA-125 in normal, hyperplastic and malignant endometrial tissue. Anticancer Research $\mathbf{2 3}$ 1075-1080.

Mylonas I, Makovitzky J, Richter DU, Jeschke U, Briese V \& Friese K 2004 Expression of the inhibin-alpha subunit in normal, hyperplastic and malignant endometrial tissue: an immunohistochemical analysis. Gynecology \& Oncology 93 92-97.

Patel JH, Loboda AP, Showe MK, Showe LC \& McMahon SB 2004 Analysis of genomic targets reveals complex functions of MYC. Nature Reviews in Cancer 4 562-568.

Powles TJ 1998 Status of antiestrogen breast cancer prevention trials. Oncology (Huntington) 12 28-31.

Ring A \& Dowsett M 2004 Mechanisms of tamoxifen resistance. Endocrine-related Cancer 11 643-658. 
Saegusa M, Hashimura M, Yoshida T \& Okayasu I 2001 beta-Catenin mutations and aberrant nuclear expression during endometrial tumorigenesis. British Journal of Cancer 84 209-217.

Schroeder JA, Adriance MC, Thompson MC, Camenisch TD \& Gendler SJ 2003 MUC1 alters beta-catenin-dependent tumor formation and promotes cellular invasion. Oncogene 22 1324-1332.

Shang Y \& Brown M 2002 Molecular determinants for the tissue specificity of SERMs. Science 295 2465-2468.

Shang Y, Hu X, DiRenzo J, Lazar MA \& Brown M 2000 Cofactor dynamics and sufficiency in estrogen receptorregulated transcription. Cell $103843-852$.

Shukla S \& Gupta S 2004 Suppression of constitutive and tumor necrosis factor alpha-induced nuclear factor (NF)-kappaB activation and induction of apoptosis by apigenin in human prostate carcinoma PC-3 cells: correlation with down-regulation of NF-kappaBresponsive genes. Clinical Cancer Research 10 3169-3178.

Siufi AA, Cotrim GDSID, De Cassia MDR, Takita LC, Rodrigues De Lima G \& Goncalves WJ 2003 Effects of tamoxifen therapy on the expression of p27 protein in the endometrium of women with primary breast cancer. International Journal of Oncology 23 1545-1551.

Smid-Koopman E, Blok LJ, Helmerhorst TJ, ChadhaAjwani S, Burger CW, Brinkmann AO \& Huikeshoven FJ 2004 Gene expression profiling in human endometrial cancer tissue samples: utility and diagnostic value. Gynecology \& Oncology 93 292-300.

Valk PJ, Verhaak RG, Beijen MA, Erpelinck CA, Barjesteh van Waalwijk van Doorn-Khosrovani S,
Boer JM, Beverloo HB, Moorhouse MJ, van der Spek PJ, Lowenberg B et al. 2004 Prognostically useful gene-expression profiles in acute myeloid leukemia. New England Journal of Medicine $\mathbf{3 5 0}$ 1617-1628.

Veronesi U, Maisonneuve P, Costa A, Sacchini V, Maltoni C, Robertson C, Rotmensz N \& Boyle P 1998 Prevention of breast cancer with tamoxifen: preliminary findings from the Italian randomised trial among hysterectomised women. Italian Tamoxifen Prevention Study. Lancet 352 93-97.

Walters RW, Freimuth P, Moninger TO, Ganske I, Zabner J \& Welsh MJ 2002 Adenovirus fiber disrupts CAR-mediated intercellular adhesion allowing virus escape. Cell 110 789-799.

Yanaihara A, Otsuka Y, Iwasaki S, Koide K, Aida T \& Okai T 2004 Comparison in gene expression of secretory human endometrium using laser microdissection. Reproductive Biology and Endocrinology 2 66-72.

Yoshida S, Harada T, Mitsunari M, Iwabe T, Sakamoto Y, Tsukihara S, Iba Y, Horie S \& Terakawa N 2004 Hepatocyte growth factor/Met system promotes endometrial and endometriotic stromal cell invasion via autocrine and paracrine pathways. Journal of Clinical Endocrinology and Metabolism 89 823-832.

Zhang D, Simmen RC, Michel FJ, Zhao G, Vale-Cruz D \& Simmen FA 2002 Secretory leukocyte protease inhibitor mediates proliferation of human endometrial epithelial cells by positive and negative regulation of growthassociated genes. Journal of Biological Chemistry 277 29999-30009. 
